\title{
Nanofibrous Spongy Microspheres Enhance Odontogenic Differentiation of Human Dental Pulp Stem Cells
}

\author{
Rong Kuang, Zhanpeng Zhang, Xiaobing Jin, Jiang Hu, Melanie J. Gupte, \\ Longxing $\mathrm{Ni}$,* and Peter X. Ma*
}

Dentin regeneration is challenging due to its complicated anatomical structure and the shortage of odontoblasts. In this study, a novel injectable cell carrier, nanofibrous spongy microspheres (NF-SMS), is developed for dentin regeneration. Biodegradable and biocompatible poly(L-lactic acid)-block-poly(Llysine) are synthesized and fabricated into NF-SMS using self-assembly and thermally induced phase separation techniques. It is hypothesized that NFSMS with interconnected pores and nanofibers can enhance the proliferation and odontogenic differentiation of human dental pulp stem cells (hDPSCs), compared to nanofibrous microspheres (NF-MS) without pore structure and conventional solid microspheres (S-MS) with neither nanofibers nor pore structure. During the first $9 \mathrm{~d}$ in culture, hDPSCs proliferate significantly faster on NF-SMS than on NF-MS or S-MS $(p<0.05)$. Following in vitro odontogenic induction, all the examined odontogenic genes (alkaline phosphatase content, osteocalcin, bone sialoprotein, collagen 1, dentin sialophosphoprotein (DSPP)), calcium content, and DSPP protein content are found significantly higher in the NF-SMS group than in the control groups. Furthermore, 6 weeks after subcutaneous injection of hDPSCs and microspheres into nude mice, histological analysis shows that NF-SMS support superior dentin-like tissue formation compared to NF-MS or S-MS. Taken together, NF-SMS have great potential as an injectable cell carrier for dentin regeneration. caries, the pulp can be exposed and damaged, requiring its removal due to the risk of pulp necrosis, ${ }^{[2]}$ periapical periodontitis, ${ }^{[3]}$ and infection of associated other tissues. ${ }^{[4]}$ Furthermore, losing pulp vitality directly affects the function of the tooth. Current dental treatments to prevent pulp damage and promote dentin repair include pulp capping and root canal therapy. ${ }^{[5]}$ However, these treatments can cause tooth discoloration, increased brittleness, and ultimately tooth loss. ${ }^{[6]}$ Therefore, a novel dentin repair therapy is highly desired.

Tissue engineering technology provides a new approach to dentin repair, which aims to regenerate dentin to replace the impaired dental tissue..$^{[7-9]}$ The invested cell sources for dentin regeneration include dental pulp stem cells (DPSCs), stem cells from the apical part of the papilla (SCAPs), and stem cells from human exfoliated deciduous teeth (SHED). ${ }^{[7,10]}$ Particularly, DPSCs are an attractive dental stem cell source because of their high availability and high potential for dentin repair. ${ }^{[1]}$ However, all of these adherent stem cells require beneficial scaffolding for the cells to attach, proliferate and differentiate. Therefore, scaffolds play a key role in dentin tissue engineering, which should be designed with a high porosity and interconnected pore network to facilitate cell seeding, adhesion, extracellular matrix (ECM) secretion, and progressively the mineralized dentin regeneration. ${ }^{[12,13]}$ Naturally derived materials such as collagen, chitosan, and hyaluronic acid have previously been investigated for cell attachment and guidance. ${ }^{[14,15]}$ However, the physical properties of these natural hydrogel materials
Dr. R. Kuang, Prof. L. Ni

State Key Laboratory of Military Stomatology

Department of Operative Dentistry and Endodontics

School of Stomatology

The Fourth Military Medical University

Xi'an 710032, P. R. China

E-mail: nilx2007@gmail.com

Dr. R. Kuang, Dr. X. Jin, Dr. J. Hu, Prof. P. X. Ma

Department of Biologic and Materials Sciences

University of Michigan

Ann Arbor, MI 48109, USA

E-mail: mapx@umich.edu
Dr. Z. Zhang, Dr. M. J. Gupte, Prof. P. X. Ma

Department of Biomedical Engineering

University of Michigan

Ann Arbor, MI 48109, USA

Prof P. X. Ma

Macromolecular Science and Engineering Center

University of Michigan

Ann Arbor, MI 48109, USA

Prof. P. X. Ma

Department of Materials Science and Engineering

University of Michigan

Ann Arbor, MI 48109, USA

DOI: 10.1002/adhm.201500308 

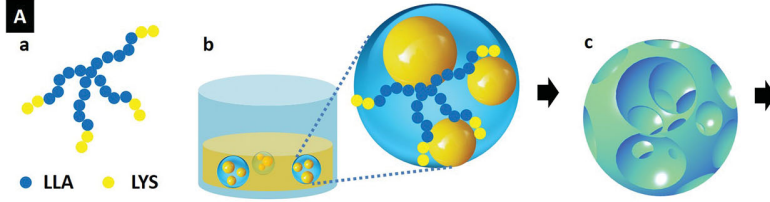

d

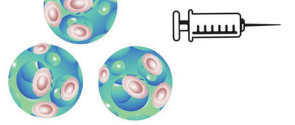

B
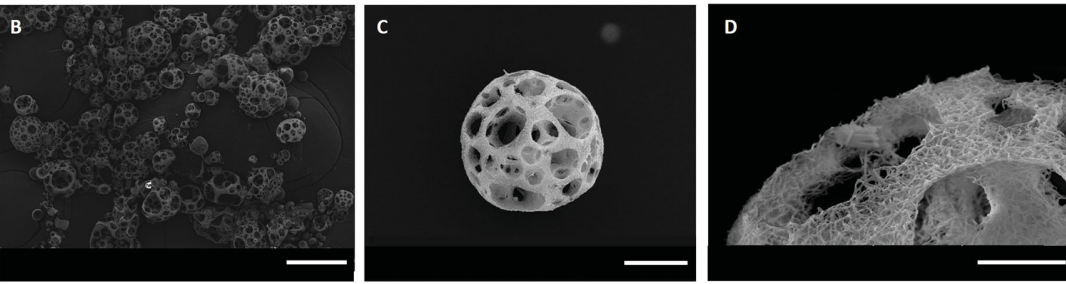

Figure 1. A) A schematic illustration of the fabrication of NF-SMS for stem cell delivery through injection. a) SS-PLLA- $b$-PLYS; b) emulsions self-assembled from SS-PLLA- $b$-PLYS, with one polymer solution droplet containing multiple glycerol domains; c) NF-SMS were obtained after phase separation and freeze drying; d) the porous structure of NF-SMS allows efficient cell loading and delivery through injection. B-D) Characterization of NF-SMS. B) SEM image of NF-SMS at low magnification. C) SEM image of representative NF-SMS, showing the interconnected porous structure with a pore diameter around 10-20 $\mu \mathrm{m}$. D) High-magnification image of (C) showing the NF architecture of the NF-SMS with an average fiber diameter of about $160 \mathrm{~nm}$. Scale bars: B) $100 \mu \mathrm{m}$, C) $20 \mu \mathrm{m}$, and D) $10 \mu \mathrm{m}$.
(PLLA-b-PLYS) copolymer using an emulsification method recently developed in our lab. ${ }^{20]}$ Of note, the introduction of PLYS blocks into the polymer drove the polymer self-assembly into NF-SMS, while homopolymer star-shaped PLLA can only form nanofibrous hollow microspheres without an interconnected porous structure. NF-SMS with a diameter ranging from 30 to $60 \mu \mathrm{m}$ were chosen for dentin regeneration using DPSCs in this work. The highly porous structure with interconnected pores had an average opening size of 10-20 $\mu \mathrm{m}$ throughout the microspheres and a porosity of about 95\% (Figure 1B,C). The nanofibers in the microsphere walls have an average fiber diameter of about $160 \mathrm{~nm}$ (Figure 1D). To investigate the effect of the microsphere architecture, NF-MS without pores and S-MS without nanofibers of the same diameter were fabricated as controls. may not be desirable because they have poor mechanical properties and uncontrolled degradation kinetics. Therefore, synthetic polymers with tailored degradation rates and high processability are increasingly used in tissue engineering. ${ }^{[16]}$

In our previous study, a 3D macroporous and nanofibrous poly(L-lactic acid) (PLLA) scaffold with a high porosity and wellinterconnected pores was found to enhance human dental pulp stem cells (hDPSCs) proliferation and odontogenic differentiation. ${ }^{[8,17]}$ While this porous scaffold successfully regenerated dentin, the quality of the tissue leaves much to be desired. In addition, an injectable formulation is preferable in the dental clinical setting due to the small defect size, irregular defect shape, and desired short treatment time. To this end, injectable hydrogels, hydroxyapatite particles, and human dentin matrix ${ }^{[18]}$ have been explored for dentin regeneration. However, these materials have various limitations, ${ }^{[19]}$ such as low porosity, undesired degradability, high brittleness, or low availability, etc. We took a new approach and developed novel nanofibrous spongy microspheres. This was the first time to use the novel injectable microspheres as a cell carrier to regenerate dentin (Figure 1A). We hypothesized that these nanofibrous spongy microspheres (NF-SMS) would better support dentin regeneration than their nonporous nanofibrous counterparts (NF-MS) or solid microspheres (S-MS) without the nanofibrous feature. Specifically, the potential of NF-SMS in dentin regeneration was explored by evaluating the effect of NF-SMS on proliferation and odontogenic differentiation of hDPSCs and dentin formation, in comparison with NF-MS and S-MS.

\section{Results}

\subsection{Structure of Nanofibrous Spongy Microspheres}

Nanofibrous spongy microspheres were successfully fabricated from a star-shaped poly(L-lactic acid)-block-polylysine

\subsection{NF-SMS Enhanced hDPSCs Proliferation In Vitro}

After seeding hDPSCs on NF-SMS, NF-MS, and S-MS for $24 \mathrm{~h}$, the interactions of hDPSCs with the different microsphere architectures were examined under scanning electromicroscope (SEM) and laser scanning confocal microscope (LSCM). The SEM results revealed that hDPSCs adhered to all of the three types of microspheres (Figure 2A,C,E). On the NF-SMS, the cells attached not only to the surface but also to the interior areas and with abundant cellular processes (Figure 2A). Additionally, many cells can attach to a single NF-SMS, shown by fluorescent 4',6-diamidino-2-phenylindole (DAPI) staining of cell nuclei (Figure 2B). On the NF-MS, hDPSCs only attached to the surface and with fewer cellular processes (Figure 2C,D). In contrast, on the S-MS, the cells greatly spread out on the surface into a nonphysiological 2D morphology with limited cellular processes (Figure 2E,F). Furthermore, hDPSCs proliferated faster on NF-SMS than on the other two types of microspheres during the first $9 \mathrm{~d}$ of in vitro culture (Figure 3).

\subsection{NF-SMS Enhanced the Odontogenic Differentiation of hDPSCs In Vitro}

The alkaline phosphatase (ALP) content of hDPSCs peaked at $7 \mathrm{~d}$ and decreased by $14 \mathrm{~d}$ in all three groups. At $7 \mathrm{~d}$, the NF-SMS group had significantly higher ALP content $(p<0.05)$ than the NF-MS group, while no significant statistical difference was found at 3 or $14 \mathrm{~d}$ (Figure 4A). The NF-SMS group also had significantly higher ALP content $(p<0.05)$ than the S-MS group at all tested time points of 3, 7, and $14 \mathrm{~d}$ (Figure 4A). In addition, calcium was extracted at 2 and 4 weeks and the quantities were normalized against the sample weights. NF-SMS group had significantly higher calcium content $(p<0.05)$ than other two groups at both 2 and 4 weeks (Figure 4B). 

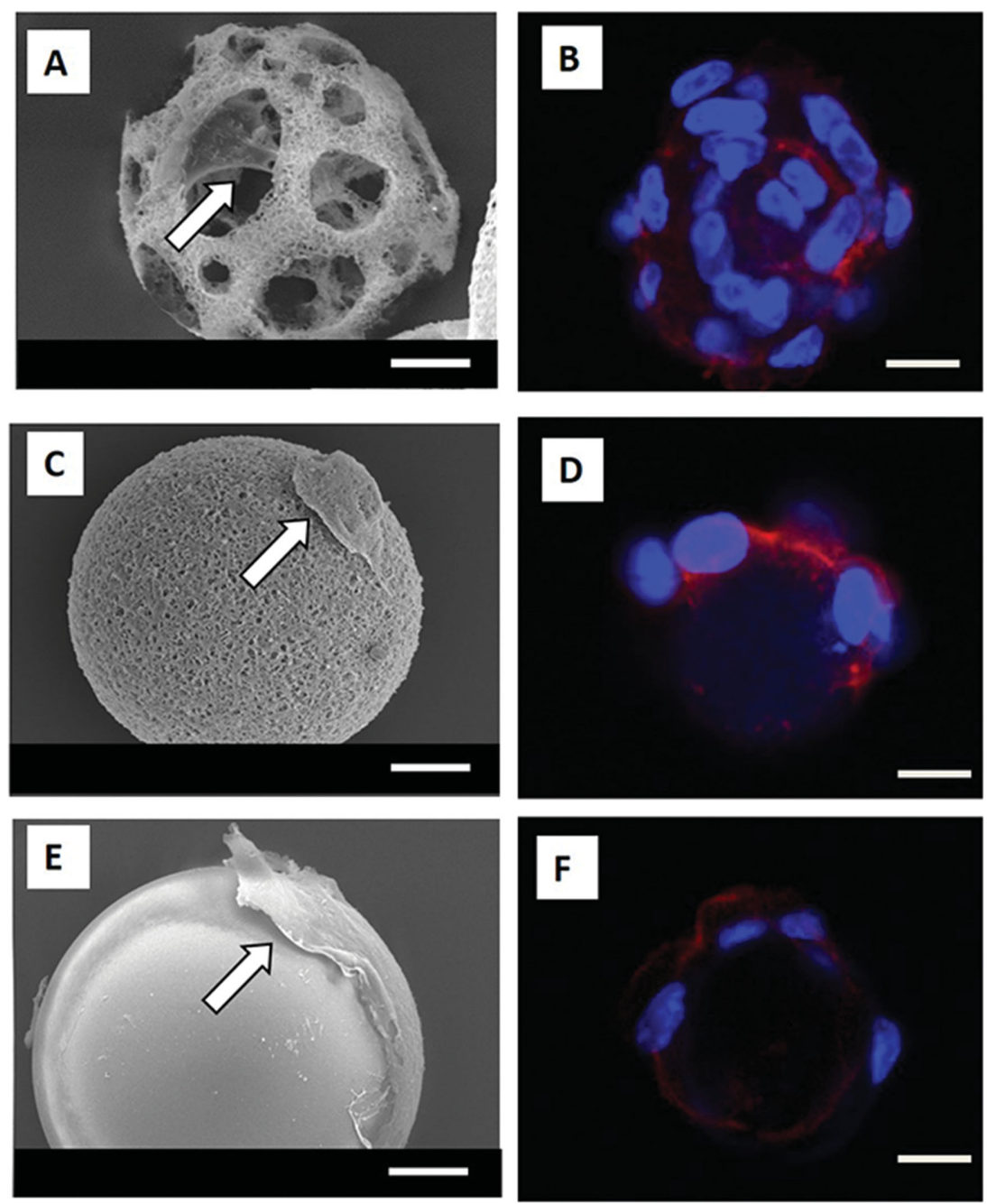

Figure 2. Interactions of hDPSCs with the three types of microspheres. A) SEM image of hDPSCs seeded on NF-SMS for $24 \mathrm{~h}$, showing the attachment of cells on both the surface and interior of the spheres, with abundant cellular processes. B) LSCM image of DPSCs seeded on NF-SMS for $24 \mathrm{~h}$, showing cells attached on the surface and inner pores of NF-SMS. C) SEM image of hDPSCs seeded on NF-MS for $24 \mathrm{~h}$, showing the cells only attached on the surface of NF-MS, with fewer cellular processes. D) LSCM image of hDPSCs seeded on NF-MS for $24 \mathrm{~h}$, showing cells attached only on the surface of NF-MS. E) SEM image of DPSCs seeded on $\mathrm{S}$-MS for $24 \mathrm{~h}$, showing spread cells on the surface of the microspheres with limited cellular processes. F) LSCM image of hDPSCs seeded on S-MS for $24 \mathrm{~h}$, showing fewer cells attached on the surface of the S-MS. Blue: nuclei and red: F-actin. Scale bars: $10 \mu \mathrm{m}$. White arrows indicate the cells attached on the microspheres.

Odontogenic related genes of hDPSCs including bone sialoprotein (BSP), osteocalcin (OCN), collagen 1 (COL1), and dentin sialophosphoprotein (DSPP) were detected by real-time polymerase chain reaction (PCR). The expression levels of BSP, OCN, and COL1 in hDPSCs increased with culture time from 2 to 4 weeks on all of three types of microspheres (Figure 4C-E). The BSP, OCN, COL1, and DSPP expression of hDPSCs on NF-SMS were significantly higher compared to the S-MS group at both time points $(p<0.05)$. Compared with NF-MS group, BSP and COL1 expression of hDPSCs on NF-SMS were significantly higher at both 2 and 4 weeks, while the OCN gene expression of hDPSCs on NF-SMS was significantly higher only at 4 weeks. The DSPP gene expression in NF-SMS group was significantly higher than that in NF-MS group $(p<0.05)$ (Figure 4F). Furthermore, immunofluorescence staining was used to visualize DSPP expression after odontogenic induction for 4 weeks (Figure 5). Strong positive DSPP staining was detected on the surface and interior of the NF-SMS (Figure 5, top row), while DSPP was only detected on the surface of the NF-MS (Figure 5, middle row) and was negligible in the S-MS group (Figure 5, bottom row).

\subsection{NF-SMS Enhanced the Odontogenic Differentiation of hDPSCs In Vivo}

Histological results showed that neo tissue developed on all three types of microspheres after subcutaneous injection for 6 weeks. By gross view, the NF-SMS and NF-MS groups had larger tissue volumes than the S-MS group (Figure 6A-C). H-E staining showed significant tissue formation in the NF-SMS group, with a large number of cells evenly distributed throughout the tissue and NF-SMS almost completely degraded by this time (Figure 6D). The tissue formed on the NF-MS had fewer cells than the NF-SMS group, and the NF-MS did not completely degrade within the 6 weeks (Figure 6E). In the S-MS group, many S-MS occupied the space of the tissue with the fewest cells surrounding the microspheres (Figure 6F). The dentin-like tissue formed in vivo on the three different types of microspheres was analyzed with von Kossa staining for mineralization (Figure 6G-I) and DSPP immunohistochemical staining for dentin matrix deposition (Figure 6J-L). NF-SMS supported the odontogenic differentiation of hDPSCs and dentin tissue formation with a significant level of mineralization (Figure 6G) and strong DSPP staining (Figure 6J). The NF-MS group contained less mineralized tissue with weak von Kossa staining (Figure 6H) and marginal DSPP staining (Figure 6K), while S-MS supported only fibrous tissue formation with negligible mineralization (Figure 6I) and minimal DSPP staining (Figure 6L). Collectively, NF-SMS greatly enhanced the odontogenic differentiation of hDPSCs and dentinogenesis in vivo in the ectopic injection model.

\section{Discussion}

Dentin regeneration has the potential to benefit the entire adult population and about $60 \%-70 \%$ of the pediatric population because of the prevalence of dental caries. ${ }^{[1]}$ Nevertheless, dentin matrix is only secreted by odontoblasts, a terminal differentiated cell type, which is hard to isolate and has very limited 


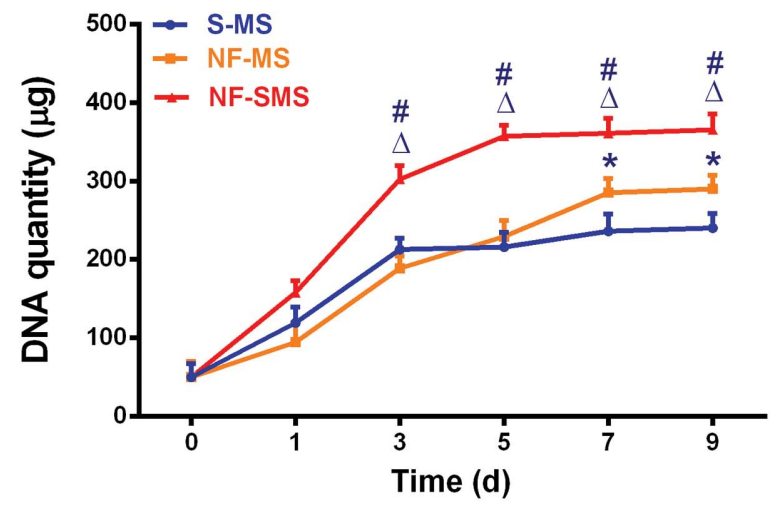

Figure 3. NF-SMS enhanced hDPSCs proliferation in vitro. Growth curve of hDPSCs cultured on NF-SMS, NF-MS, and S-MS, measured by quantifying the DNA content at $0,1,3,5,7$, and $9 \mathrm{~d}$, respectively $(n=3) . \Delta$ : $p<0.05$ NF-SMS versus NF-MS, \#: $p 0.05$ NF-SMS versus S-MS, $*$ $p<0.05$ NF-MS versus S-MS.

expansion capacity. Therefore, stem cells that can differentiate down the odontogenic lineage are highly sought after cell sources for dentin regeneration. Despite the significant recent advances in stem cell biology, controlling the differentiation direction of stem cells remains a daunting challenge. Hence, porous scaffolds could be explored as a biomimetic microenvironment to preferentially guide stem cell differentiation directions. ${ }^{[8,16,17,21]}$ This was the first time we used the novel NF-SMS to enhance the odontogenic differentiation of hDPSCs and thereby to facilitate dentin regeneration.

Scaffolds should possess at least three essential functions. First, a scaffold must provide a 3D template to guide tissue shape formation. Second, a scaffold should provide a suitable surface for cell attachment, proliferation, and differentiation. ${ }^{[15]}$ Third, a scaffold should provide an open porous network for cell seeding/migration, nutrient/waste transport, and needed cell-cell interactions. ${ }^{[13,22]}$

For dental tissue engineering, an injectable scaffold is more preferable than an implantable 3D bulk scaffold because dental defects are often small and have irregular shapes. Porous microspheres are explored as injectable cell carriers for tissue repair, ${ }^{[23]}$ but no previous technology allows the presentation of desirable nanofibrous feature on porous microspheres. In this study, novel injectable microspheres (NF-SMS) were used as a cell carrier to regenerate dentin, which can easily fill small and irregular shaped defects. Nanofibers in a 3D bulk scaffold mimic the fibrous architecture of ECM and were shown to promote hDPSCs proliferation and differentiation when compared to nonnanofibrous materials. ${ }^{[8,24]}$ Consistently, the biomimetic nanofibrous feature and the porous structure of the NF-SMS significantly improved hDPSCs attachment, proliferation, and their odontogenic differentiation over the control microspheres. Because cells in suspension have an average diameter of around $10 \mu \mathrm{m}$, the diameter of pores in the NF-SMS was designed to be $10-20 \mu \mathrm{m}$ to facilitate the cell infiltration into the internal space. Accordingly, more cells attached on the NF-SMS both on the outer surface and inside the pores than on the NF-MS without pores. In addition to the improved cell seeding efficiency and proliferation rate, the highly interconnected pores in the NF-SMS also enabled the needed cell-cell interactions to enhance the DSPP expression and odontoblast maturation.

It has been reported that when hDPSCs are seeded on various scaffolds, such as collagen sponge, porous ceramics, or fibrous titanium meshes, they often form a tissue that more resembles a connective tissue than a dentin-like tissue, ${ }^{[25]} \mathrm{dem}$ onstrating the significant challenge of inducing the osteogenic differentiation of hDPSCs. In this study, we examined several important odontogenic markers in hDPSC cultures on NF-SMS, NF-MS, and S-MS. ALP regulates organic and inorganic phosphate metabolism, acts as a plasma membrane transporter for inorganic phosphates, ${ }^{[26]}$ and is widely used as an early marker of osteogenic differentiation. The ALP content in the NF-SMS group was significantly higher than that in either NF-MS group or S-MS group at $7 \mathrm{~d}$ of induction. OCN plays an important role in matrix deposition and biomineralization ${ }^{[27]}$ and therefore is widely used as a late marker of odontogenic differentiation. The OCN gene expression level in NF-SMS group was significantly higher than in either NF-MS group or S-MS group at both 2 and 4 weeks of induction. As the major noncollagenous dentinal protein, DSPP is essential for dentin mineralization ${ }^{[28]}$ and is considered to be a highly specific tissue marker for dentin. DSPP gene expression was significantly higher in NF-SMS group than in NF-MS or S-MS group at both 2 and 4 weeks of induction. DSPP protein was positively detected in NF-SMS and NF-MS cultures but was at the negligible level in S-MS culture via immunofluorescence staining. Moreover, DSPP was detected both on the outer surface and in the inner pores of the NF-SMS, while it was only detected on the outer surface of NF-MS, which indicates the higher potential of using NF-SMS to regenerate denser dentin than NF-MS. All of these in vitro results indicate that the NF-SMS enhance the odontogenic differentiation of hDPSCs compared to the two control cell carriers, NF-MS and S-MS.

The tissue formation 6 weeks after subcutaneous injection of the microsphere-hDPSC complexes into mice supported our hypothesis that NF-SMS can better support dentin-like tissue regeneration than nonporous NF-MS and S-MS. The tissue volume of NF-SMS group was the largest among the three groups, while the tissue volume of S-MS group was the smallest. Most NF-SMS degraded after 6 weeks of implantation and had the highest cellularity. NF-MS degraded slower and a significant amount of them remained in the neo tissue at this time. S-MS did not show obvious degradation and remained essentially intact at this time. The porosity and degradation rate of the cell carriers thus appeared to play important roles in tissue formation. First, the porous structure of NF-SMS at the micrometer scale could provide more space for cells to penetrate the scaffold and form bigger and denser tissues, as compared to NF-MS and S-MS. Second, the surface to volume ratios of NF-SMS and NF-MS $\left(\approx 120 \mathrm{~m}^{2} \mathrm{~g}^{-1}\right)$ are substantially higher than that of the S-MS $\left(\approx 1 \mathrm{~m}^{2} \mathrm{~g}^{-1}\right)$ due to the nanofibrous structure, which makes NF-SMS and NF-MS degrade significantly faster than S-MS. In addition, the porous structure at the micrometer scale in the NF-SMS further increases the porosity and lowers the density of the microspheres $\left(\approx 0.02 \mathrm{~g} \mathrm{~cm}^{-3}\right)$ as compared to NF-MS $\left(\approx 0.13 \mathrm{~g} \mathrm{~cm}^{-3}\right)$, resulting in an even faster degradation rate. Therefore, NF-SMS can degrade quickly to relinquish the space to the expanded cells 

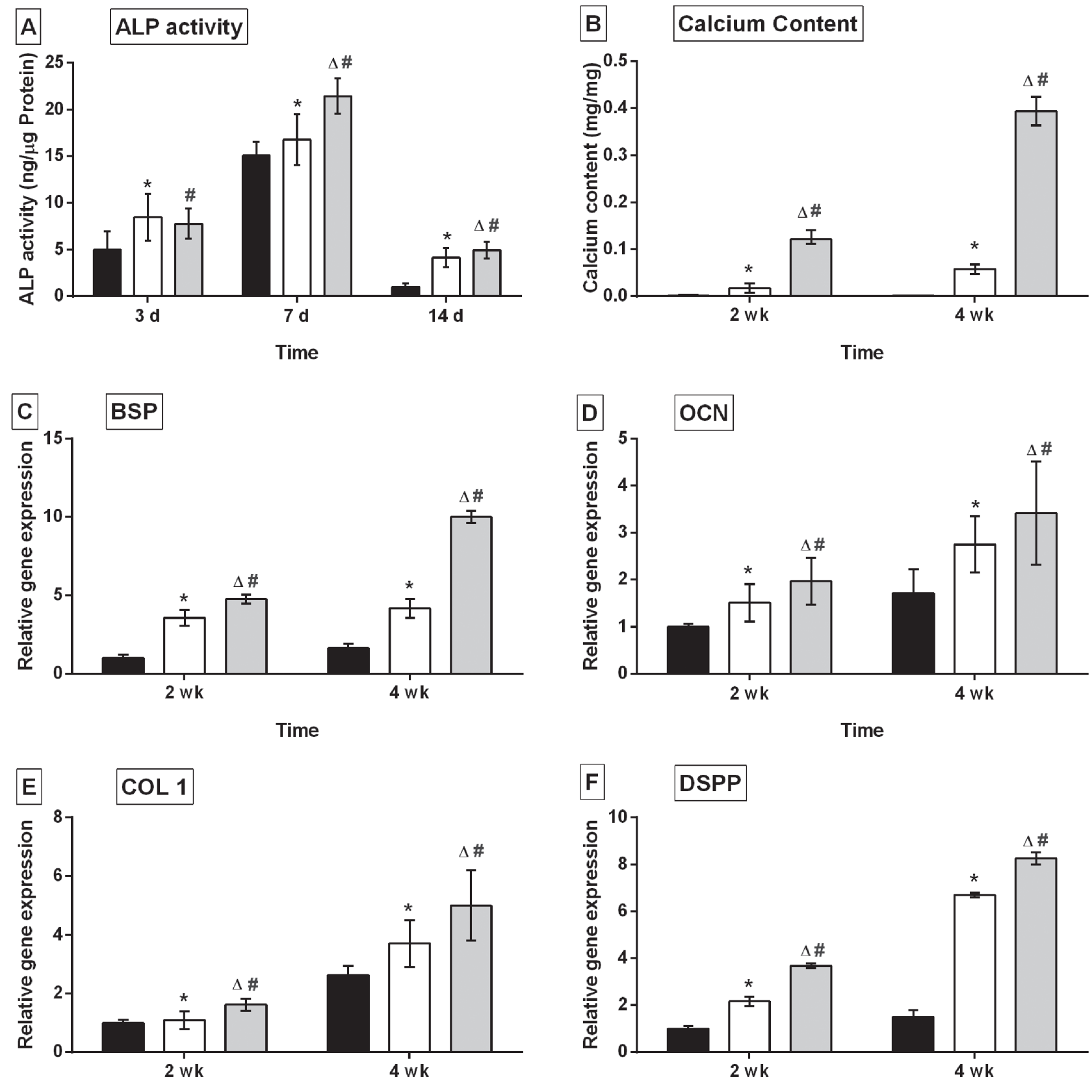

Time

Time

Figure 4. NF-SMS enhanced the odontogenic differentiation of hDPSCs in vitro. A) ALP content of hDPSCs cultured on NF-SMS, NF-MS, and S-MS after 3, 7, and $14 \mathrm{~d}$ of induction. B) Calcium content of cell/microsphere complexes after 2 and 4 weeks of induction. C-F) Real-time PCR quantification of the expression levels of odontogenic genes of hDPSCs on the NF-SMS, NF-MS, and S-MS after 2 and 4 weeks of induction. The gene expressions of BSP C), OCN D), COL1 E), and DSPP F) were higher on NF-SMS than on NF-MS and S-MS at the indicated time points $(n=3)$. $\Delta: p<0.05$ NF-SMS versus NF-MS, \#:p<0.05 NF-SMS versus S-MS, *: $p<0.05$ NF-MS versus S-MS.

and regenerated tissue. Furthermore, NF-SMS supported more mineralized tissue formation, likely benefited at least in part from the highest DSPP content among the three groups. Taken together, NF-SMS with nanofibrous and a porous structure significantly promoted odontogenic differentiation of hDPSCs and dentin tissue formation.
In conclusion, NF-SMS were used to regenerate dentin for the first time in this work. The injectable NF-SMS provided a beneficial microenvironment for hDPSC proliferation, odontogenic differentiation, and dentin tissue regeneration. These results clearly demonstrate that NF-SMS are a promising injectable cell carrier with high clinical potential for dentin repair. 

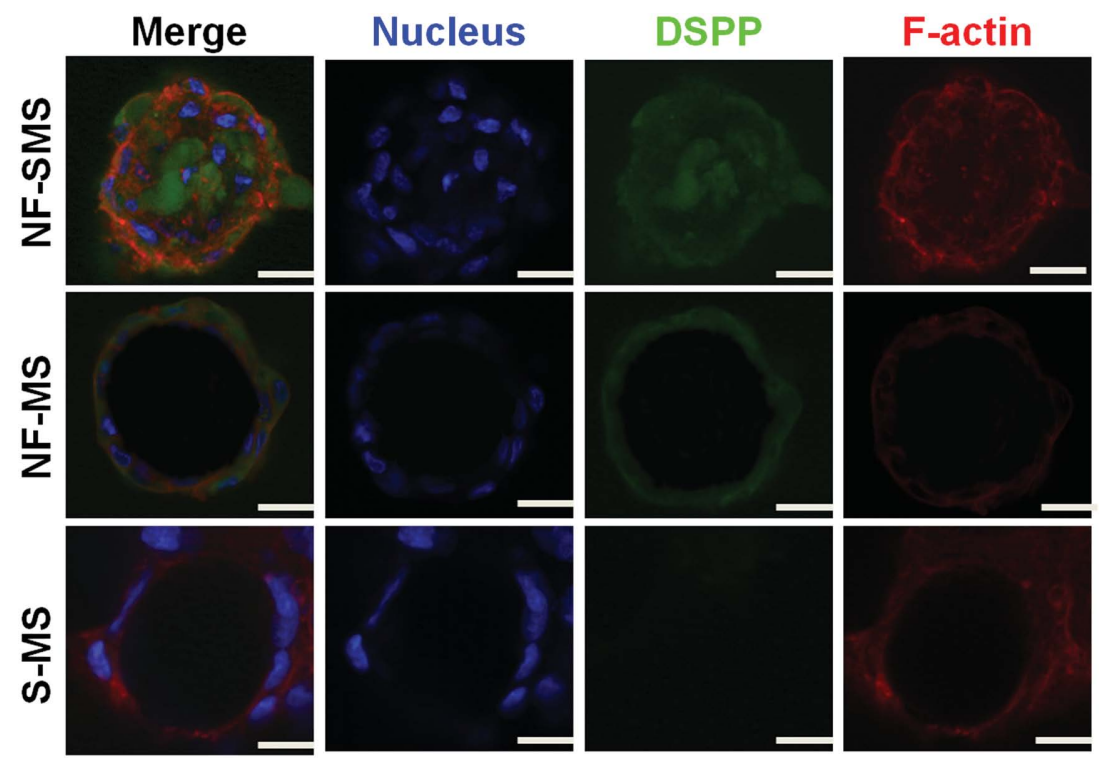

Figure 5. NF-SMS enhanced DSPP protein expression of hDPSCs in vitro. DSPP immunofluorescence staining of hDPSCs on NF-SMS, NF-MS, and S-MS after odontogenic induction for 4 weeks. Blue: nuclei; green: DSPP; and red: F-actin. The images show that DSPP was detected not only on the surface of NF-SMS but also inside its inner pores; DSPP was only detected on the surface of NF-MS; DSPP was negative in S-MS group. Scale bars: $10 \mu \mathrm{m}$.

Future studies will focus on dentin repair and regeneration in situ in a tooth defect model.

\section{Experimental Section}

Materials: Poly(amidoamine) dendrimer with 16 hydroxyl end groups (PAMAM-OH, generation 2) was purchased from Sigma-Aldrich (St. Louis, MO) and dried at $50^{\circ} \mathrm{C}$ in a vacuum oven for $24 \mathrm{~h}$ before use. L-Lactide (Sigma-Aldrich) was recrystallized from toluene twice. $\mathrm{N}_{6}$ Carbobenzyloxy-L-lysine (Chem-Impex International, Wood Dale, IL), $1,1^{\prime}$-carbonyldiimidazole (Sigma-Aldrich), $N, N^{\prime}$-dicyclohexylcarbodiimide (DCC, Sigma-Aldrich), N-tert-butoxycarbonyl-L-phenylalanine (Phe$\mathrm{N}_{\mathrm{BOC}}$, Sigma-Aldrich), and Tin(II) 2-ethylhexanoate $\left(\mathrm{Sn}(\mathrm{Oct})_{2}, 95 \%\right.$, Sigma-Aldrich) were used as received. Dimethyl sulfoxide was refluxed over $\mathrm{CaH}_{2}$ and distilled under nitrogen. Tetrahydrofuran (THF) was dried and distilled in the presence of sodium immediately before use. Trifluoroacetic acid, hydrogen bromide, and acetic acid were purchased from Sigma-Aldrich and used without purification.

Synthesis of Star-Shaped Poly(L-lactic-acid)-co-Poly(L-lysine) (PLLA-bPLYS): The star-shaped PLLA-b-PLYS was synthesized using a method modified from one reported previously.[20] Briefly, star-shaped PLLA was polymerized from monomer L-lactide through ring-opening polymerization using dendrimer PAMAM (generation 2 with hydroxyl end groups). Star-shaped PLLA was then reacted with $N$-tert-butoxycarbonylL-phenylalanin/ $N, N^{\prime}$-dicyclohexylcarbodiimide, followed by trifluoroacetic acid treatment to convert the hydroxyls on each end of the polymer arm into amino groups. SS-PLLA- $\mathrm{NH}_{2}$ was employed for the polymerization of lysine $\mathrm{N}$-carboxyanhydrides with carbobenzyloxy protecting groups, which were then removed by $\mathrm{HBr} / \mathrm{HAc}$ solution after the polymerization. The synthesized star-shaped block copolymer PLLA-b-PLYS was stored in vacuum at room temperature.

Fabrication of NF-SMS: A reversed emulsification method was used to generate the NF-SMS from star-shaped PLLA-b-PLYS. PLLA was dissolved in THF at $50{ }^{\circ} \mathrm{C}$ with a concentration of $2.0 \%(\mathrm{w} / \mathrm{v})$. Under rigorous mechanical stirring (speed 7, MAXIMA, Fisher Scientific Inc.,
Pittsburgh, PA), the polymer solution was quickly added into glycerol prewarmed at $50{ }^{\circ} \mathrm{C}$ and the stirring continued for 5 more min. The mixture was then quickly poured into liquid nitrogen. After $10 \mathrm{~min}$, ice/water mixture was added to exchange solvent for $24 \mathrm{~h}$. The spheres were then sieved and washed with distilled water for three times to remove glycerol residue. The spheres were then lyophilized for $2 \mathrm{~d}$. The surface areas were calculated from Brunauer-Emmett-Teller plot of adsorption/desorption isotherm using adsorption points in the $P / P_{0}$ range of $0.1-0.3$ (BELSORP-mini analysis software). The porosity of microspheres was calculated from $\varepsilon=1-D_{\mathrm{p}} / D_{0}$, where $D_{\mathrm{p}}$ was the overall density of the polymer microsphere aggregation and $D_{0}$ was the density of the solid polymer. $D_{\mathrm{p}}$ was determined by $D_{\mathrm{p}}=4 \mathrm{~m} /\left(\pi d^{2} h\right)$, where $m, d$, and $h$ were the mass, the diameter, and the thickness of the microspheres in a disc shaped container, respectively.

Cell Isolation and Culture: Dental pulp tissues were harvested from extracted healthy human third molars collected from patients 16-20 years of age at the University of Michigan, School of Dentistry. The protocol was approved by the Institutional Review Board at the University of Michigan. hDPSCs were isolated from molars according to the protocol reported previously. ${ }^{[1]}$ The pulp tissue was gently separated from the crown and roots and then digested in a solution of $3 \mathrm{mg} \mathrm{mL}^{-1}$ collagenase type I (Worthington Biochem, Freehold, NJ) and $4 \mathrm{mg} \mathrm{mL}^{-1}$ dispase (Boehringer Mannheim, Ingelheim am Rhein, German) for $1 \mathrm{~h}$ at $37^{\circ} \mathrm{C}$. Single-cell suspensions were obtained by passing the cells through a $70 \mu \mathrm{m}$ strainer. Single-cell suspensions $\left(0.01\right.$ to $\left.1 \times 10^{5} \mathrm{well}^{-1}\right)$ of dental pulp were seeded into six-well plates with alpha modification of Eagle's medium ( $\alpha$-MEM) (Invitrogen, Carlsbad, CA) supplemented with $15 \%$ fetal bovine serum (FBS) (Invitrogen), $100 \times 10^{-3} \mathrm{M}$ L-ascorbic acid 2-phosphate (Sigma-Aldrich), $2 \times 10^{-3} \mathrm{M} \mathrm{L-glutamine}$ and $1 \%$ penicillinstreptomycin (Invitrogen), and then incubated at $37^{\circ} \mathrm{C}$ in $5 \% \mathrm{CO}_{2}$. After the first passage, the hDPSCs were maintained in $\alpha$-MEM supplemented with 10\% FBS (Invitrogen) and 1\% penicillin-streptomycin (Invitrogen). The medium was changed every two days and hDPSCs of passages 3-6 were used in the following studies.

Cell Seeding on Microspheres: The microspheres were soaked in $70 \%$ ethanol to prewet for $30 \mathrm{~min}$ and then were exchanged with phosphatebuffered saline (PBS) for three times (30 min each). The microspheres were then washed with $\alpha$-MEM containing $10 \%$ FBS for $30 \mathrm{~min} .8 \times 10^{6}$ cells and $4 \times 10^{5}$ microspheres were mixed in $2 \mathrm{~mL}$ medium in a $15 \mathrm{~mL}$ centrifuge tube on orbital shaker at $12 \mathrm{rpm}$ for $4 \mathrm{~h}$. Then the mixture was transferred into a spinner flask (Wheaton industries Inc., Millville, NJ) and maintained in $80 \mathrm{~mL}$ culture medium with stirring at $80 \mathrm{rpm}$. For odontogenic differentiation assay, after $24 \mathrm{~h}$ of cell seeding the normal culture medium was replaced with odontogenic induction medium, which contained $\alpha$-MEM supplemented with $10 \% \mathrm{FBS}, 50 \mu \mathrm{g} \mathrm{mL} \mathrm{m}^{-1}$ ascorbic acid, $5 \times 10^{-3} \mathrm{M} \beta$-glycerophosphate, and $10^{-7} \mathrm{M}$ dexamethasone. The medium was changed every two days.

Scanning Electron Microscopy (SEM) Observation: The hDPSCs cultured on different microspheres were rinsed in PBS once, fixed in $2.5 \%$ glutaraldehyde, and postfixed in $1 \%$ osmium tetroxide for $1 \mathrm{~h}$. Samples were dehydrated in a series of increasing concentrations of ethanol and hexamethyldisilizane. The samples were then sputter coated with gold using a sputter coater (SPI-MODULE, West Chester, PA) and observed under a scanning electron microscope (Philips XL30 FEG, Amsterdam, Holland) at $10 \mathrm{kV}$.

DNA Quantification Assay: A DNA quantification kit (Sigma) was used according to the manufacturer's protocol. Briefly, the cell-sphere complexes were rinsed with PBS once, homogenized in 1 $1 \times$ DNA assay buffer and equal volume of a lysis buffer (Sigma), and then incubated at 

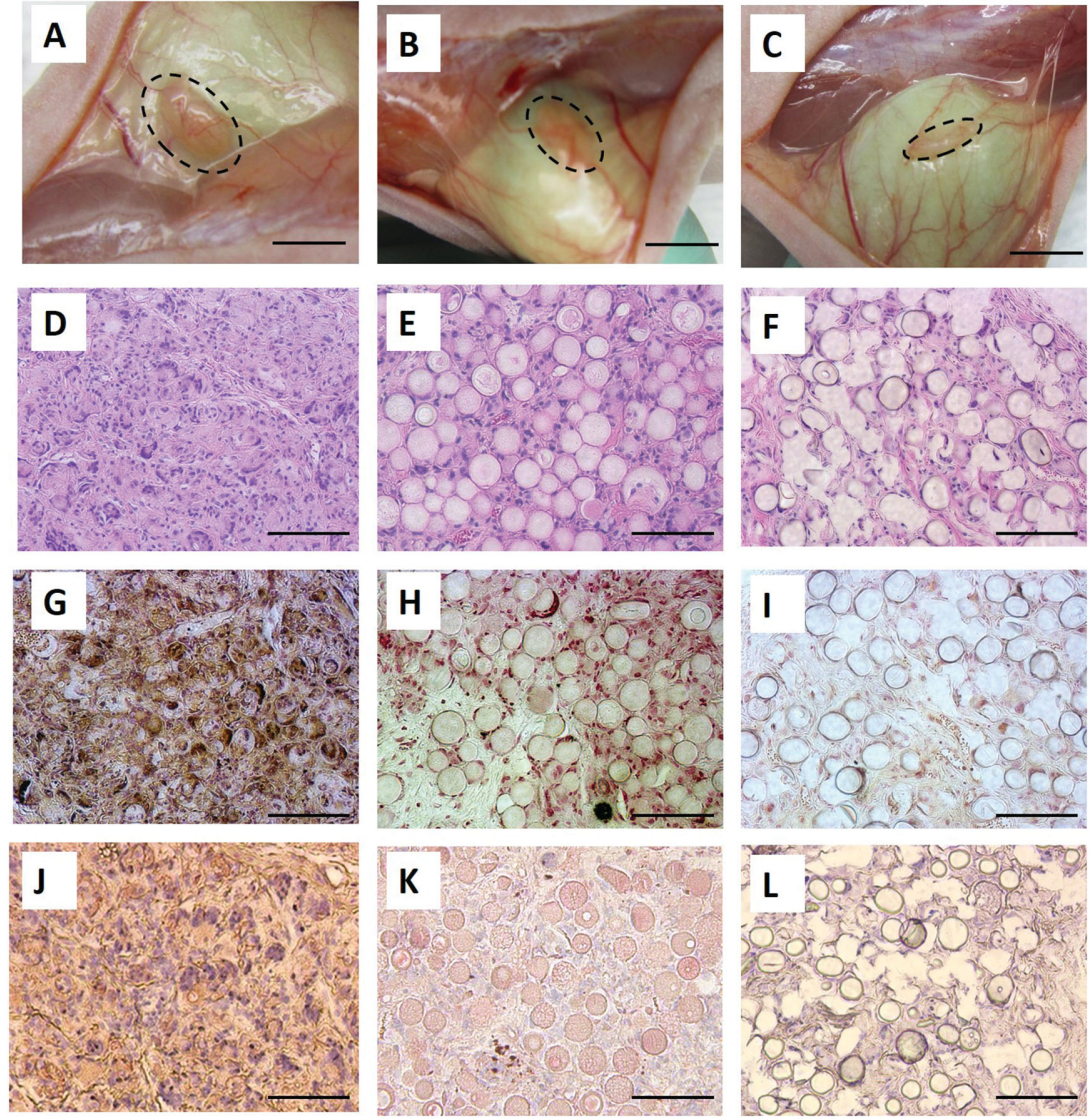

Figure 6. NF-SMS enhanced hDPSCs odontogenic differentiation in vivo. The DPSCs cultured on NF-SMS, NF-MS, and S-MS were preinduced with odontogenic medium for $7 \mathrm{~d}$, then the cell/microsphere complexes were subcutaneously injected on the back of nude mice and harvested 6 weeks later. Gross views of neo tissue in NF-SMS group A), NF-MS group B), and $\mathrm{S}-\mathrm{MS}$ group $\mathrm{C}$ ). The dashed line circles indicated the contours of the neo tissues. $\mathrm{H}$-E staining D-F) showed tissue formation in all groups. Most NF-SMS degraded and there were abundant cells in the neo tissue D). NF-MS partially degraded and there were fewer cells in the neo tissues than in the NF-SMS group but more than in the S-MS group E). S-MS remained persistent and there were fewest cells in the neo tissue of S-MS group. von-Kossa staining showed significantly high mineralization in NF-SMS group (G) than in NF-MS group $(\mathrm{H})$ and S-MS group (I). IHC staining showed high level of DSPP in NF-SMS group (J), low level of DSPP in NF-MS group $(K)$, and negligible level of DSPP in S-MS group (L). Scale bars: (A-C): $2 \mathrm{~mm}$; (D-L): $100 \mu \mathrm{m}$.

$37^{\circ} \mathrm{C}$ for $1 \mathrm{~h}$. Cell lysate was centrifuged at $5000 \mathrm{~g}$ at room temperature for $3 \mathrm{~min}$. The supernatant was collected for DNA content determination using fluorescence assay with Hoechst 33258 dye (Sigma) under a Varioskan Flash multimode reader (Thermo Scientific, Wyman Street Waltham, MA). The excitation wavelength was $360 \mathrm{~nm}$ and emission wavelength was $460 \mathrm{~nm}$.

ALP Content Quantification: ALP content was detected using a SensoLyte pNPP Alkaline Phosphatase Assay Kit (AnaSpec, Fremont, CA) according to the manufacturer's protocol. Briefly, cells on the microspheres were rinsed with $1 \times$ buffer, homogenized in $300 \mu \mathrm{L}$ lysis buffer (supplied with the kit) on ice for $10 \mathrm{~min}$. Lysates were centrifuged at $2500 \mathrm{~g}$ at $4{ }^{\circ} \mathrm{C}$ for $10 \mathrm{~min}$. Supernatant was collected for ALP assay using $p$-nitrophenyl phosphate as a phosphatase substrate and alkaline phosphatase supplied by the kit as a standard. The absorbance was measured at $405 \mathrm{~nm}$ with a Varioskan Flash multimode reader (Thermo Fisher Scientific). The amount of ALP in the cells was normalized against total protein content. The total protein amount was quantified using a Micro BCA protein assay kit (Thermo scientific, Rockford, IL) following the protocol of the manufacturer.
Calcium Content Quantification: Samples were weighed, rinsed for three times with distilled water, and then homogenized in $200 \mu \mathrm{L} 1 \mathrm{~m}$ acetic acid. The lysates were incubated overnight at $4{ }^{\circ} \mathrm{C}$ to extract calcium. The total calcium content of each sample was determined by 0 -cresolphthaleincomplexone method following the manufacturer's instructions (Calcium liquiColor, Stanbio Laboratory, Boerne, TX). The calcium amount was normalized by sample weight in $\mathrm{mg}$.

Real-Time PCR: Total RNA was extracted using RNA Mini kit (Qiagen, Valencia, CA) and the first-strand CDNA was reverse transcribed using SuperScript II Reverse Transcriptase (Invitrogen). Quantitative real-time PCR was performed using ready-made Taqman primers (Life Technologies, calsbad, CA) for three odontogenic marker genes: BSP (Hs00173720_ml), OCN (Hs01587814_g1), and type I collagen (COL1A1) (Hs00164004_m1) and normalized by reduced glyceraldehydephosphate dehydrogenase (Hs99999905_ml). Alternately, dentin sialophosphoprotein (DSPP) gene expression quantification was performed with sybergreen primers synthesized by Life Technologies, the sequence was TTAAATGCCAGTGGAACCAT (5'-3', forward), and ATTCCCTTCTCCCTTCTCAC (5'-3', reverse). Real time PCR was performed with a ViiA 7 Real-Time PCR System (Applied Biosystems).

Inmmunofluorescence Staining and LSCM Imaging: For F-actin staining, after seeding for $24 \mathrm{~h}$, the hDPSCs on NF-SMS, NF-MS, and S-MS were rinsed with PBS, fixed with $4 \%$ formaldehyde solution and treated with $0.1 \%$ Triton X-100 for 3 min, blocked with PBS containing 2\% bovine serum albumin (BSA) at room temperature for $20 \mathrm{~min}$. The samples were incubated with Alexa Fluor 555 phalloidin (Life Technologies) for $30 \mathrm{~min}$, rinsed for three times with PBS, transferred into a eight-well chamber, and mounted with Vectashield mounting medium containing DAPI (Vector Laboratories, Burlingame, CA). The samples were observed under a laser scanning confocal microscope (Nikon TS-100, Tokyo, Japan). For immunofluorescence staining of DSPP, the hDPSCmicrosphere samples were collected after 4 weeks of odontogenic induction. Samples were washed, fixed, treated with Triton X-100, and blocked with BSA as described above. The samples were then incubated with 1:100 DSPP primary antibody (Santa Cruz Biotechnology, Santa Cruz, CA) overnight at $4{ }^{\circ} \mathrm{C}$, rinsed with PBS, and subsequently incubated with fluorescein-conjugated secondary donkey antimouse IgG antibody (Santa Cruz Biotechnology Inc.) at room temperature for $45 \mathrm{~min}$. For double-staining with F-actin, samples were further incubated with Alexa Fluor 555 phalloidin as described above, mounted with Vectashield mounting medium containing DAPI and observed under a LSCM (Nikon TS-100). All images were taken at a cross-section of the samples.

Subcutaneous Injection: The animal surgery protocol was approved by the University Committee on Use and Care of Animals (UCUCA) at the University of Michigan. Six nude mice (nu/nu) 6-8 weeks old (Charles River Laboratories, Wilmington, MA) were used in this study. Surgery was performed under anesthesia by general inhalation of $2 \%$ isoflurane. The hDPSCs and microspheres were mixed at $2 \times 10^{6}$ cells and $10^{5}$ spheres per injection in $2 \mathrm{~mL}$ culture medium for $4 \mathrm{~h}$ and were cultured in spinner flasks with odontogenic medium for $7 \mathrm{~d}$ before subcutaneous injection. The cell-microsphere complexes were then centrifuged and resuspended in $1 \mathrm{~mL}$ of cell culture media. Four $100 \mu \mathrm{L}$ injections of the mixture of cells and microspheres were made subcutaneously in 
the dorsa of each nude mouse and at least six samples per group $(n=$ 6). Animals were euthanized and samples were retrieved after 6 weeks following injection. Harvested samples were immediately fixed in $10 \%$ formalin for $24 \mathrm{~h}$ and then processed for histological observation.

Histological Observation: Animals were euthanized and samples were retrieved. Harvested samples were immediately fixed in 10\% formalin for $24 \mathrm{~h}$ and processed for paraffin embedding and sectioning for hematoxylin and eosin (H\&E) staining, von-Kossa staining and immunohistological staining. For immunohistological staining of DSPP, sections were dewaxed with xylene and a series aqueous ethanol solution with decreasing concentrations, incubated with $5 \%$ pepsin (Thermo Scientific) for epitope retrieval at $37^{\circ} \mathrm{C}$ for $30 \mathrm{~min}$ and blocked with 5\% BSA for $20 \mathrm{~min}$. After rinsing with PBS, the sections were incubated with 1:50 DSPP primary antibody (Santa Cruz Biotechnology) at $4{ }^{\circ} \mathrm{C}$ overnight and stained using the Cell and Tissue Staining Kit ( $R$ \& D Systems) following the manufacturer's protocol.

Statistical Analysis: Numerical data were reported as mean \pm S.D. ( $n=3$ for in vitro samples and $n=6$ for in vivo samples). The experiments were performed twice to ensure reproducibility. To test the significance of observed differences between the study groups, Student's $t$-test was applied. A value of $p<0.05$ was considered to be statistically significant.

\section{Acknowledgements}

The authors would like to acknowledge the financial support from the National Institutes of Health (NIDCR DE015384, DE017689, and DE022327: P.X.M.), the funds for young teachers to study abroad from the School of Stomatology of FMMU (R.K.), and the predoctoral fellowship from NIDCR Training Grant 5T32DE007057 and the GAANN Fellowship (M.J.G.).

Received: April 25, 2015 Revised: June 8, 2015 Published online: July 2, 2015

[1] a) N. Joshi, S. Sujan, K. Joshi, H. Parekh, B. Dave, J. Int. Oral Health 2013, 5, 35; b) A. Kamppi, T. Tanner, J. Pakkila, P. Patinen, M. R. Jarvelin, L. Tjaderhane, V. Anttonen, Caries Res. 2013, 47, 346; c) R. Srivastava, S. K. Gupta, V. P. Mathur, A. Goswami, B. Nongkynrih, Southeast Asian J. Trop. Med. Public Health 2013, 44, 523.

[2] Y. Zheng, X. Y. Wang, Y. M. Wang, X. Y. Liu, C. M. Zhang, B. X. Hou, S. L. Wang, J. Dent. Res. 2012, 91, 676.

[3] M. Kovacevic, T. Tamarut, N. Jonjic, A. Braut, Aust. Endod. J. 2008, $34,12$.

[4] A. Hodgdon, Emerg. Med. Clin. North Am. 2013, 31, 465.

[5] G. T. Huang, Front. Biosci., Elite Ed. 2011, 3, 788.

[6] F. Fonzar, A. Fonzar, P. Buttolo, H. V. Worthington, M. Esposito, Eur. J. Oral Implantol. 2009, 2, 201.

[7] R. Alsanea, S. Ravindran, M. I. Fayad, B. R. Johnson, C. S. Wenckus, J. Hao, A. George, J. Endod. 2011, 37, 1092.

[8] J. Wang, H. Ma, X. Jin, J. Hu, X. Liu, L. Ni, P. X. Ma, Biomaterials 2011, 32, 7822.

[9] S. Eap, T. Bécavin, L. Keller, T. Kökten, F. Fioretti, J. L. Weickert, E. Deveaux, N. Benkirane-Jessel, S. Kuchler-Bopp, Adv. Healthcare Mater. 2014, 3, 386.
[10] a) G. T. Huang, Regener. Med. 2009, 4, 697; b) K. lohara, M. Nakashima, M. Ito, M. Ishikawa, A. Nakasima, A. Akamine, J. Dent. Res. 2004, 83, 590; c) W. Sonoyama, Y. Liu, D. Fang, T. Yamaza, B. M. Seo, C. Zhang, H. Liu, S. Gronthos, C. Y. Wang, S. Wang, S. Shi, PLoS One 2006, 1, e79.

[11] S. Gronthos, M. Mankani, J. Brahim, P. G. Robey, S. Shi, Proc. Natl. Acad. Sci. U.S.A. 2000, 97, 13625.

[12] a) P. X. Ma, J. W. Choi, Tissue Eng. 2001, 7, 23; b) A. Al-Abboodi, J. Fu, P. M. Doran, T. T. Y. Tan, P. P. Y. Chan, Adv. Healthcare Mater. 2014, 3, 725.

[13] S. W. Choi, Y. Zhang, M. R. Macewan, Y. Xia, Adv. Healthcare Mater. 2013, 2, 145.

[14] a) J. Aigner, J. Tegeler, P. Hutzler, D. Campoccia, A. Pavesio, C. Hammer, E. Kastenbauer, A. Naumann, J. Biomed. Mater. Res. 1998, 42, 172; b) B. Chevallay, D. Herbage, Med. Biol. Eng. Comput. $2000,38,211$; c) C. Zhu, D. Fan, Z. Duan, W. Xue, L. Shang, F. Chen, Y. Luo, J. Biomed. Mater. Res. A 2009, 89, 829.

[15] J. J. Rice, M. M. Martino, L. De Laporte, F. Tortelli, P. S. Briquez, J. A. Hubbell, Adv. Healthcare Mater. 2013, 2, 57.

[16] P. X. Ma, Adv. Drug Delivery Rev. 2008, 60, 184.

[17] J. Wang, X. Liu, X. Jin, H. Ma, J. Hu, L. Ni, P. X. Ma, Acta Biomater. 2010, 6, 3856.

[18] a) Y. Ando, M. J. Honda, H. Ohshima, A. Tonomura, T. Ohara, T. Itaya, H. Kagami, M. Ueda, Nagoya J. Med. Sci. 2009, 71, 51; b) B. R. Coyac, F. Chicatun, B. Hoac, V. Nelea, C. Chaussain, S. N. Nazhat, M. D. McKee, J. Dent. Res. 2013, 92, 648; c) W. Guo, Y. He, X. Zhang, W. Lu, C. Wang, H. Yu, Y. Liu, Y. Li, Y. Zhou, J. Zhou, M. Zhang, Z. Deng, Y. Jin, Biomaterials 2009, 30,6708; d) B. Yang, G. Chen, J. Li, Q. Zou, D. Xie, Y. Chen, H. Wang, X. Zheng, J. Long, W. Tang, W. Guo, W. Tian, Biomaterials 2012, 33, 2449; e) R. Li, W. Guo, B. Yang, L. Guo, L. Sheng, G. Chen, Y. Li, Q. Zou, D. Xie, X. An, Y. Chen, W. Tian, Biomaterials 2011, 32, 4525; f) S. J. Park, Z. Li, I. N. Hwang, K. M. Huh, K. S. Min, J. Endod. 2013, 39, 1001.

[19] Z. Yuan, H. Nie, S. Wang, C. H. Lee, A. Li, S. Y. Fu, H. Zhou, L. Chen, J. J. Mao, Tissue Eng., Part B 2011, 17, 373.

[20] Z. Zhang, R. L. Marson, Z. Ge, S. C. Glotzer, P. X. Ma, Adv. Mater. 2015, DOI: 10.1002/adma.201501329.

[21] a) L. A. Smith, X. Liu, P. X. Ma, Soft Matter 2008, 4, 2144; b) L. A. Smith, P. X. Ma, Colloids Surf., B 2004, 39, 125.

[22] P. X. Ma, Adv. Drug Delivery Rev. 2008, 60, 184.

[23] a) J. Fang, Y. Zhang, S. Yan, Z. Liu, S. He, L. Cui, J. Yin, Acta Biomater. 2014, 10, 276; b) K. Garkhal, A. Mittal, S. Verma, N. Kumar, Polym. Adv. Technol. 2011, 22, 190; c) T. K. Kim, J. J. Yoon, D. S. Lee, T. G. Park, Biomaterials 2006, 27, 152; d) Q. Zhang, K. Tan, Z. Ye, Y. Zhang, W. Tan, M. Lang, Mater. Sci. Eng. C 2012, 32, 2589.

[24] a) T. Qu, X. Liu, J. Mater. Chem. B 2013, 1, 4764; b) X. Yang, F. Yang, X. F. Walboomers, Z. Bian, M. Fan, J. A. Jansen, J. Biomed. Mater. Res., Part A 2010, 93, 247.

[25] W. Zhang, X. F. Walboomers, T. H. van Kuppevelt, W. F. Daamen, Z. Bian, J. A. Jansen, Biomaterials 2006, 27, 5658.

[26] Q. Liu, L. Cen, S. Yin, L. Chen, G. Liu, J. Chang, L. Cui, Biomaterials 2008, 29, 4792.

[27] X. Jiang, J. Zhao, S. Wang, X. Sun, X. Zhang, J. Chen, D. L. Kaplan, Z. Zhang, Biomaterials 2009, 30, 4522.

[28] a) R. N. D'Souza, A. Cavender, G. Sunavala, J. Alvarez, T. Ohshima, A. B. Kulkarni, M. MacDougall, J. Bone Miner. Res. 1997, 12, 2040; b) J. Q. Feng, X. Luan, J. Wallace, D. Jing, T. Ohshima, A. B. Kulkarni, R. N. D'Souza, C. A. Kozak, M. MacDougall, J. Biol. Chem. 1998, 273, 9457; c) Y. Yamakoshi, J. Oral Biosci. 2008, 50, 33. 